\title{
Thermoresponsive Behavior of Magnetic Nanoparticle Complexed pNIPAm-co-AAc Microgels
}

\author{
Su-Kyoung Lee, Yongdoo Park and Jongseong Kim * \\ Department of Biomedical Engineering, College of Medicine, Korea University, 73 Inchon-ro, Seongbuk-gu, \\ Seoul 02841, Korea; switfrik@gmail.com (S.-K.L.); ydpark67@korea.ac.kr (Y.P.) \\ * Correspondence: envokim72@korea.ac.kr; Tel.: +82-2-2286-1458
}

Received: 27 September 2018; Accepted: 16 October 2018; Published: 19 October 2018

check for updates

Featured Application: This work will provide a simple route for potential applications of magnetic nanoparticle-hydrogel complex in temperature responsive behaviors. It will be worthwhile to employ this work to develop thermoresponsive magnetic resonance imaging agent and magneto-thermo induced stimulation toolkits for cell regulation.

\begin{abstract}
Characterization of responsive hydrogels and their enhancement with novel moieties have improved our understanding of functional materials. Hydrogels coupled with inorganic nanoparticles have been sought for novel types of responsive materials, but the efficient routes for the formation and the responsivity of complexed materials remain for further investigation. Here, we report that responsive poly( $\mathrm{N}$-isopropylacrylamide-co-acrylic acid) (pNIPAm-co-AAc) hydrogel microparticles (microgels) are tunable by varying composition of co-monomer and crosslinker as well as by their complexation with magnetic nanoparticles in aqueous dispersions. Our results show that the hydrodynamic diameter and thermoresponsivity of microgels are closely related with the composition of anionic co-monomer, AAc and crosslinker, $N, N^{\prime}$-Methylenebisacrylamide (BIS). As a composition of hydrogels, the higher AAc increases the swelling size of the microgels and the volume phase transition temperature (VPTT), but the higher BIS decreases the size with no apparent effect on the VPTT. When the anionic microgels are complexed with amine-modified magnetic nanoparticles (aMNP) via electrostatic interaction, the microgels decrease in diameter at $25^{\circ} \mathrm{C}$ and shift the volume phase transition temperature (VPTT) to a higher temperature. Hysteresis on the thermoresponsive behavior of microgels is also measured to validate the utility of aMNP-microgel complexation. These results suggest a simple, yet valuable route for development of advanced responsive microgels, which hints at the formation of soft nanomaterials enhanced by inorganic nanoparticles.
\end{abstract}

Keywords: microgel; magnetic nanoparticle; electrostatic complexation; thermoresponsivity

\section{Introduction}

Stimuli-responsive hydrogels, that undergo the volume phase transition in response to external stimuli, have great value in the development of biocompatible smart materials for use in biosensors [1-5], artificial tissues [6], and many other utilities [2,4,7]. The responsivity can be carefully designed to cause the swelling/deswelling of the hydrogels and most common cases are temperature and $\mathrm{pH}$ responsive behaviors [8-11]. To achieve such responsivity, poly( $N$-isopropylacrylamide) (pNIPAm) and acrylic acid (AAc) are widely used due to the lower critical solution temperature (LCST) and the $\mathrm{pK}_{\mathrm{a}}$, respectively $[12,13]$. Above the LCST, the polymer network is favorable to be collapsed by chain-chain interactions, while above the $\mathrm{pK}_{\mathrm{a}}$ the network tends to swell due to Coulombic repulsion among the deprotonated acid groups [14-16]. Colloidal hydrogel particles (microgels) are commonly prepared by aqueous free-radical precipitation polymerization due to the low polydispersity and 
accurate size control $[13,17]$. These microgels have been extensively investigated for their fast response rate and nanoscale structure in comparison to those of bulk hydrogels $[18,19]$. The development of multi-responsive hydrogels often requires different kinds of monomers for copolymerization, and even post-modifications by conjugating interesting molecules to the hydrogels [20,21]. Therefore, efficient methods for the formation of such advanced hydrogels are highly desired.

Nanoparticles including gold nanoparticles (AuNPs) and magnetic nanoparticles (MNPs) have been widely applied in the development of functional nanosystems for optical modulators [5,22], biosensors [23,24], imaging agents [25], and biological interfacing technology [26]. This suggests that complexation of the nanoparticles with responsive hydrogels allows the development of multipotent materials in a wide range of applications [27-32]. For instance, AuNPs and MNPs are coupled with hydrogels via DNA hybridization, which shows photothermal and magnetic-field responsive activities, respectively $[27,28]$. In addition, copolymerization in which AuNPs and MNPs are used as a seed material has been used to yield hybrid nanoparticle-hydrogels in core-shell structure. Although this method allows for many interesting applications of the nanoparticle-hydrogels, it also requires a careful optimization for synthetic procedures [29-32]. Thus, there is a need for simple, fast, yet useful hybridization methods for the nanoparticle-hydrogel complexation, while retaining the hydrogel's responsivity. In this study, we aim to demonstrate that the pNIPAm-co-AAc microgels are aptly complexed with MNP, and the hybridized MNP-microgels show the characteristic thermoresponsivity in a repeatable manner. Our finding is distinguished from previous studies that utilize copolymerization of MNP-hydrogels by its simplicity and rapidity, allowing responsive pNIPAm-co-AAc microgels to acquire additional merits of magnetic nanoparticles for magneto-induced applications.

\section{Materials and Methods}

\subsection{Materials}

All reagents were obtained from Sigma-Aldrich (Saint Louis, MO, USA) unless otherwise specified. The monomer $N$-isopropylacrylamide (NIPAm), comonomer acrylic acid (AAc), cross-linker $N, N^{\prime}$-methylene(bisacrylamide) (BIS), ammonium persulfate (APS), and sodium dodecyl sulfate (SDS) were used as received. All water was deionized to a resistance of at least $18 \mathrm{M} \Omega$ (Milli-Q Integral system, Merck Millipore, Darmstadt, Germany), and then filtered through a $0.2 \mu \mathrm{m}$ filter for particulate removal. Zinc-doped iron oxide magnetic nanoparticles $(\mathrm{ZnFeO})$ coated with amine-terminated silica were gifted from Cheon group (Yonsei University, Seoul, Korea) [33].

\subsection{Microgel Synthesis}

All microgels were synthesized via aqueous free-radical precipitation polymerization, as previously described [13,34]. The syntheses were carried out by fixing the total monomer concentration constant at $70 \mathrm{mM}$ while varying the AAc mole percent of $5 \%, 10 \%$, and $20 \%$ with $2 \%$ fixed BIS mole percent and the BIS mole percent of $0 \%, 2 \%$, and $5 \%$ with $10 \%$ fixed AAc mole percent that also accordingly adjusted the NIPAm mole percent. For instance, mole percent of NIPAm is $90 \%$, $88 \%$, and $85 \%$, for $0 \%$ BIS $/ 10 \%$ AAc, $2 \%$ BIS $/ 10 \%$ AAc, and $5 \%$ BIS $/ 10 \%$ AAc microgels, respectively. Microgel synthesis was performed in a three-neck, $100 \mathrm{~mL}$ round-bottom flask. Fifty milliliters of a filtered ( $0.2 \mu \mathrm{m}$ filter, Pall Gelman Metricel, New York, NY, USA), aqueous solution of NIPAm, BIS, and the surfactant SDS ( $28.8 \mathrm{mg}, 1 \mathrm{mM}$ final concentration) were added into the reactor. The solution was heated to $\sim 70{ }^{\circ} \mathrm{C}$ while degassing with $\mathrm{N}_{2}$ and stirring with magnetic bar for $\sim 1 \mathrm{~h}$. After $1 \mathrm{~h}$, AAc was added to the flask, yielding the total monomer concentration of $70 \mathrm{mM}$. At $10 \mathrm{~min}$ after the addition of AAc, polymerization was initiated by injection of $1.0 \mathrm{~mL}$ of an APS solution $(45.64 \mathrm{mg}$, $2 \mathrm{mM}$ ). The reaction was terminated after an additional $6 \mathrm{~h}$ of heating at $\sim 70{ }^{\circ} \mathrm{C}$ and stirring while degassing by a constant flow of $\mathrm{N}_{2}$ gas. Following synthesis, the microgels were purified by using dynamic dialysis system (100 kD, Spectrum, Waltham, MA, USA). 


\subsection{MNP Synthesis}

For the current experiments, zinc-doped iron oxide magnetic nanoparticles (MNPs) were synthesized following the previous reports [33,35,36]. In brief, $\mathrm{Fe}(\mathrm{acac})_{3}(353 \mathrm{mg}), \mathrm{FeCl}_{2}(40 \mathrm{mg})$ and $\mathrm{ZnCl}_{2}(30 \mathrm{mg})$ were dissolved in octyl ether with organic surfactants (oleic acid and oleylamine). The mixture was placed in a $50 \mathrm{~mL}$ three-neck round-bottom flask and heated at $300{ }^{\circ} \mathrm{C}$ for $1 \mathrm{~h}$. Then, the reactor was cooled down to room temperature. The products were isolated by centrifugation after adding ethanol and the centrifuged magnetic nanoparticles are re-dispersed in toluene. For the amine functionalization, the MNPs are first coated by silica shell via a standard water-in-oil microemulsion sol-gel approach and the silica surface is further functionalized by aminopropyltrimethoxysilane (APTMS) [36]. The size and shape of amine-modified magnetic nanoparticles (aMNPs) were estimated by imaging using TEM (JEM-2100, JEOL, Tokyo, Japan) (Figure 1). Zeta potential value of aMNPs was measured $(19 \mathrm{mV})$ and the hydrodynamic size by dynamic light scattering (DLS) is $21 \mathrm{~nm}$ with polydispersity index (PDI) of 0.1 .

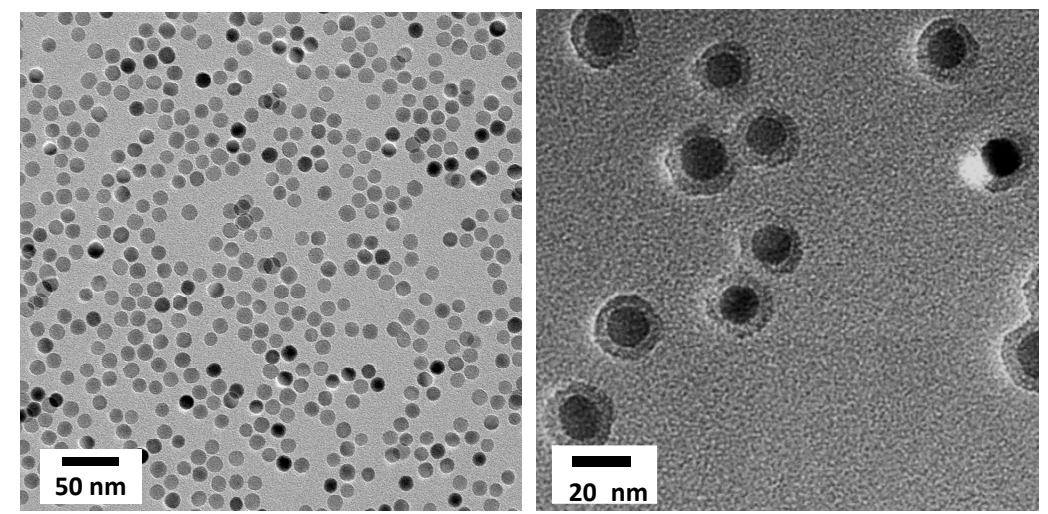

Figure 1. TEM image of $\mathrm{Zn}_{0.4} \mathrm{Fe}_{2.6} \mathrm{O}_{4}$ nanoparticles (left panel) and silica coated $\mathrm{Zn}_{0.4} \mathrm{Fe}_{2.6} \mathrm{O}_{4}$ nanoparticles (right panel). The average sizes of the nanoparticles are $13 \mathrm{~nm} \pm 1.1 \mathrm{~nm}$ and $21 \mathrm{~nm} \pm$ $1.5 \mathrm{~nm}$, respectively.

\subsection{Dynamic Light Scattering (DLS) Analysis}

The microgel was titrated by adding aliquots of $0.6 \mu \mathrm{g}$ of aMNP in solution to a dilute solution of microgels $(10 \mu \mathrm{L}$ of purified microgels diluted to $1 \mathrm{~mL})$ at $\mathrm{pH} 6.5$ ( 0.001 ionic strength) contained in a plastic cuvette. Each microgel solution was prepared before each measurement. The radius and thermoresponsive behavior of the microgels following titration was measured using Dynamic Light Scattering instrument (DLS, Zetasizer Nano ZSP, Malvern Panalytical, Malvern, UK). The radius value was an average of 12 individual radius measurements using a $5 \mathrm{~s}$ integration time for each measurement. For volume phase transition temperature (VPTT) measurements, the microgels were either first titrated with a given amount of aMNP or not. The solution was heated at an interval of $1{ }^{\circ} \mathrm{C}$ and the microgel size was determined by DLS. At each temperature, 5 consecutive runs of a sample were performed where each run was composed of 12 individual radius measurements using a $5 \mathrm{~s}$ integration time for each measurement. The error bars were estimated by the 5 consecutive runs of the same sample.

\subsection{Transmission Electron Microscopy (TEM) Analysis}

The microgel was complexed with aMNP in solution. The image of aMNP-microgels were analyzed by using a transmission electron microscope (TEM, JEM-2100, JEOL, Tokyo, Japan).

\section{Results and Discussions}

The responsive hydrogel nanoparticles are prepared by aqueous free-radical precipitation copolymerization of $\mathrm{N}$-isopropylacrylamide (NIPAm) and acrylic acid (AAc) with the use of the 
cross-linker, $N, N^{\prime}$-Methylenebisacrylamide (BIS). Microgels are hydrogel particles that are in a range of sub-micron size and with high water content in their polymeric networks. PNIPAm-co-AAc microgels are responsive to external stimuli such as temperature and $\mathrm{pH}$, resulting in the decrease or increase of the hydrodynamic radius of microgels, as shown in Figure 2a,b. In a previous study, we report that pNIPAm-co-AAc microgels are complexed with poly(allylaminehydrochloride) (PAH) via ion pairing, which shows a decrease or increase of the microgel's hydrodynamic size mainly due to the crosslinking and charge shielding effects [37]. Thus, we hypothesize that pNIPAm-co-AAc microgels are able to form the complex structure with amine-functionalized magnetic nanoparticles (aMNPs) in similar mechanisms, which provides an easy and practical method for preparation of hybrid MNP-microgels, as shown in Figure 2c,d, while retaining the thermoresponsivity. Since TEM imaging has been widely used to visualize magnetic nanoparticles, we utilize TEM analysis which displays the image of aMNP incorporated pNIPAm-co-AAc microgels, as shown in Figure 3. In addition, zeta potential and DLS values are measured to confirm the aMNP-microgel complexation, which shows the increase of the surface charge and the decrease of hydrodynamic diameter upon addition of cationic aMNP into anionic microgel solution, as shown in Figure 4.

a

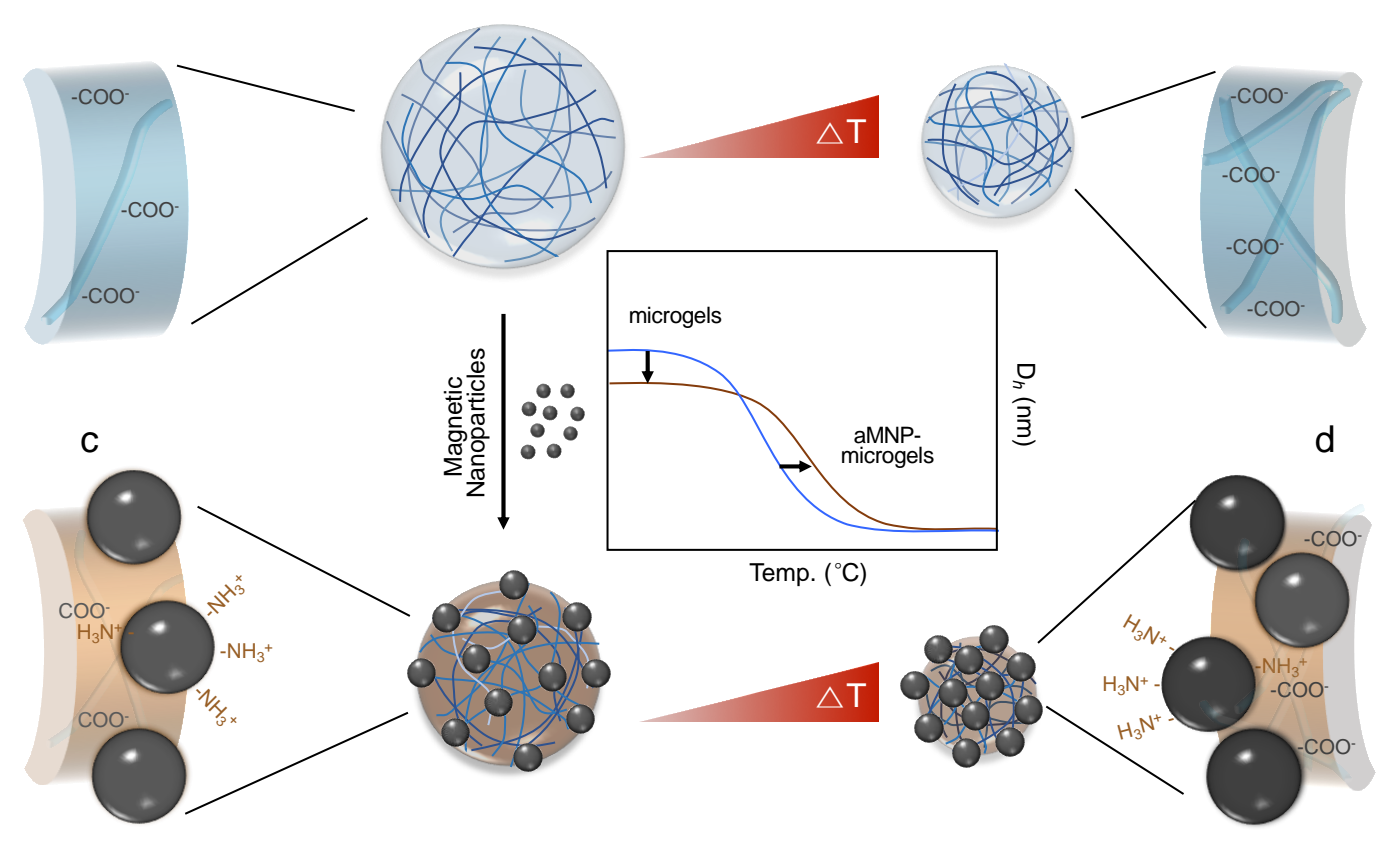

Figure 2. pNIPAm-co-AAc microgel (a) and its thermoresponsive behavior (b). Amine-functionalized magnetic nanoparticle (aMNP) enhanced microgel via electrostatic coupling (c) and its temperature dependent volume change $(\mathbf{d})$.
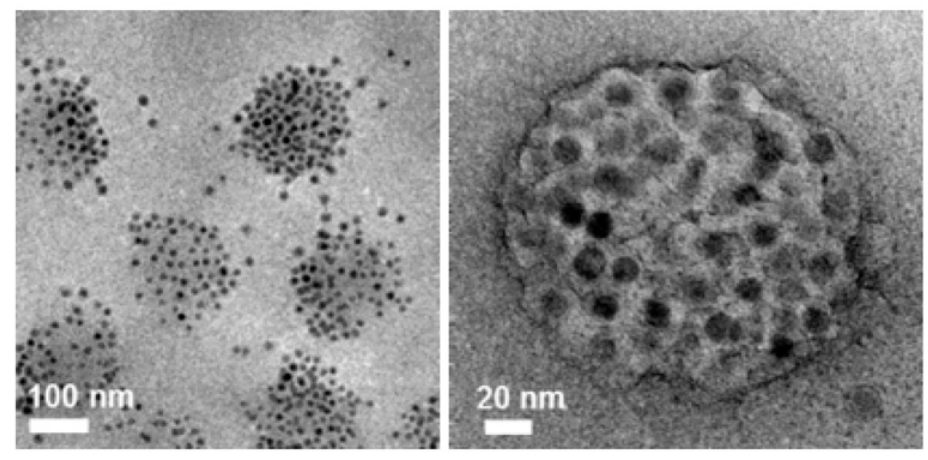

Figure 3. TEM images of aMNP complexed pNIPAm-co-AAc microgel. Low magnification (left) and high magnification (right). Note that dark circles represent aMNP incorporated to microgels. 


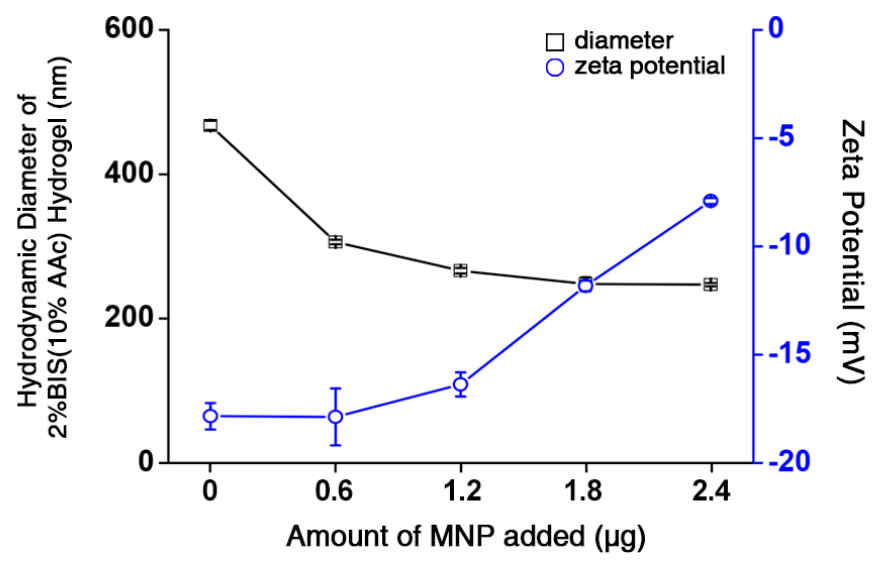

Figure 4. The zeta potential value and the hydrodynamic size for $10 \%$ AAc (acrylic acid), $2 \%$ BIS $\left(N, N^{\prime}\right.$-Methylenebisacrylamide) microgels upon $0.6,1.2,1.8$, and $2.4 \mu \mathrm{g}$ of aMNP additions. Note that zeta potential values of aMNP and microgels were measured as $19 \mathrm{mV}$ and $-18 \mathrm{mV}$, respectively.

To characterize how the pNIPAm-co-AAc microgels respond to aMNPs, we measure the hydrodynamic diameter (Dh) of the microgels upon the addition of aMNPs in aqueous solutions, as shown in Figure 5. The use of dynamic light scattering (DLS) allows the determination of the average $\mathrm{Dh}$, which shows the decrease of each Dh for various microgels, regardless of the AAc and BIS content in the microgels. In the case of different AAc content, the microgel size is decreased with positive correlation upon the addition of aMNPs. For instance, the diameter of microgels decreases from 630,470 , and $410 \mathrm{~nm}$ to 250,250 , and $300 \mathrm{~nm}$ for $20 \%, 10 \%$, and $5 \%$ of AAc, respectively, as shown in Figure 5a. It is reasonable to consider the fact that higher content of AAc yields the larger Dh of microgels mainly due to Coulombic swelling providing room for higher deswelling in which the microgel charge is compensated by positive charge of aMNPs. In contrast, the higher content of BIS shows the smaller Dh of the microgels as measured by 760, 470, and $430 \mathrm{~nm}$, respectively, while the acrylic acid ratio is constant at $10 \%$, as shown in Figure $5 \mathrm{~b}$. Their response to addition of aMNPs is inversely correlated with BIS content in the microgels. It is interesting to note that the microgels with $0 \%$ BIS dramatically change in the $\mathrm{Dh}(760$ to $350 \mathrm{~nm})$ after initial titration with aMNPs and then keep the size with no further response to aMNPs. This is partially explained by the fact that crosslinker free microgels are highly deformable by eternal stimuli [38], resulting in such abrupt change in the hydrodynamic diameter for $0 \%$ BIS microgels. The microgels with $2 \%$ and $5 \%$ BIS also decrease in the size upon addition of aMNPs and their reactivity is lower than that of $0 \%$ BIS microgels.

a

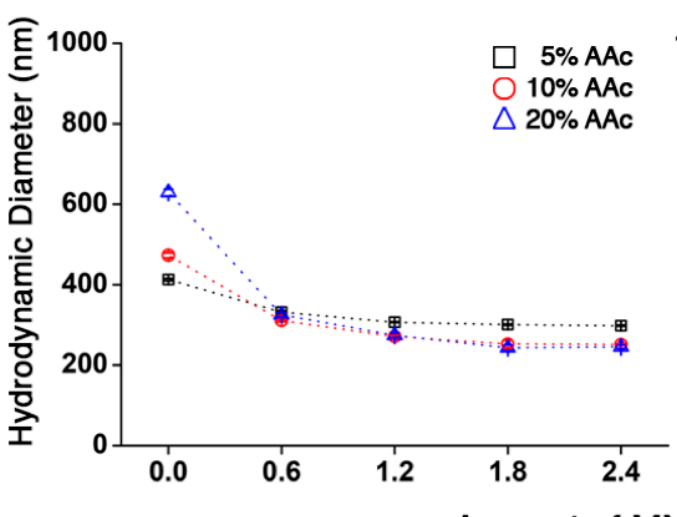

b

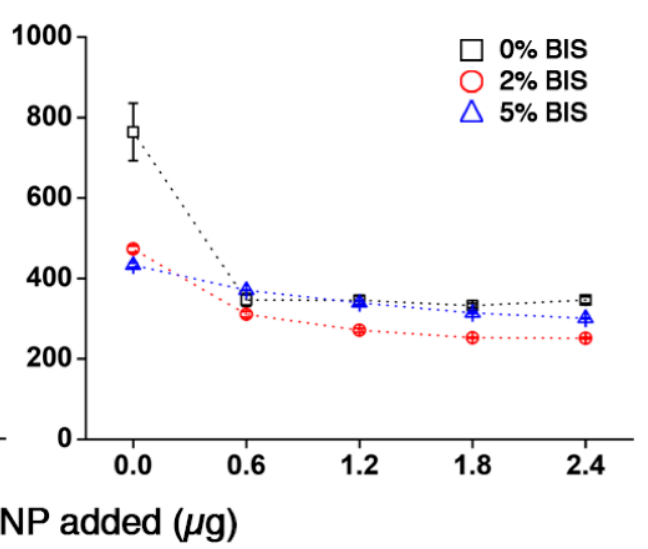

Figure 5. Hydrodynamic diameter of $5 \%(\square), 10 \%(\bigcirc)$, and $20 \%(\triangle)$ AAc $(\mathbf{a})$ or $0 \%(\square), 2 \%(\bigcirc)$, and $5 \%$ $(\triangle)$ BIS microgels $(\mathbf{b})$ upon the addition of $0.6 \mu \mathrm{g}$ of aMNP aliquots. The hydrodynamic diameter of microgels measured by dynamic light scattering (DLS) at $25^{\circ} \mathrm{C}$. 
Thermoresponsivity of the microgels is evaluated by measuring the Dh at $25^{\circ} \mathrm{C}$ and $50{ }^{\circ} \mathrm{C}$ five times, repetitively. The microgels with various AAc content $(5 \%, 10 \%, 20 \%)$ show the decreased $\mathrm{Dh}$ at $50{ }^{\circ} \mathrm{C}$, the temperature above the LCST, and their size is recovered by cooling at $25{ }^{\circ} \mathrm{C}$. Such thermoresponsivity is consistently observed for the remaining cycles for all microgels, as shown in Figure 6a, and the Dh changes increase with the AAc content of microgels. We then test the effect of aMNP complexation with microgels on their thermoresponsivity, which illustrates repeated Dh changes in the cycles of temperature, as shown in Figure $6 \mathrm{~b}$. It is worthwhile to note that the thermoresponsivity of aMNP-microgels remains for the all cycles, while their initial size is smaller than those of the uncomplexed microgels. This finding is informative when the stimuli-responsive microgels are tailored for future applications. For instance, to design more sensitive microgels for nanoparticle binding, the higher AAc composition would be beneficial. This also suggests that electrostatic complexation of microgels with aMNP is an efficient route to prepare thermoresponsive MNP-microgels for various applications.

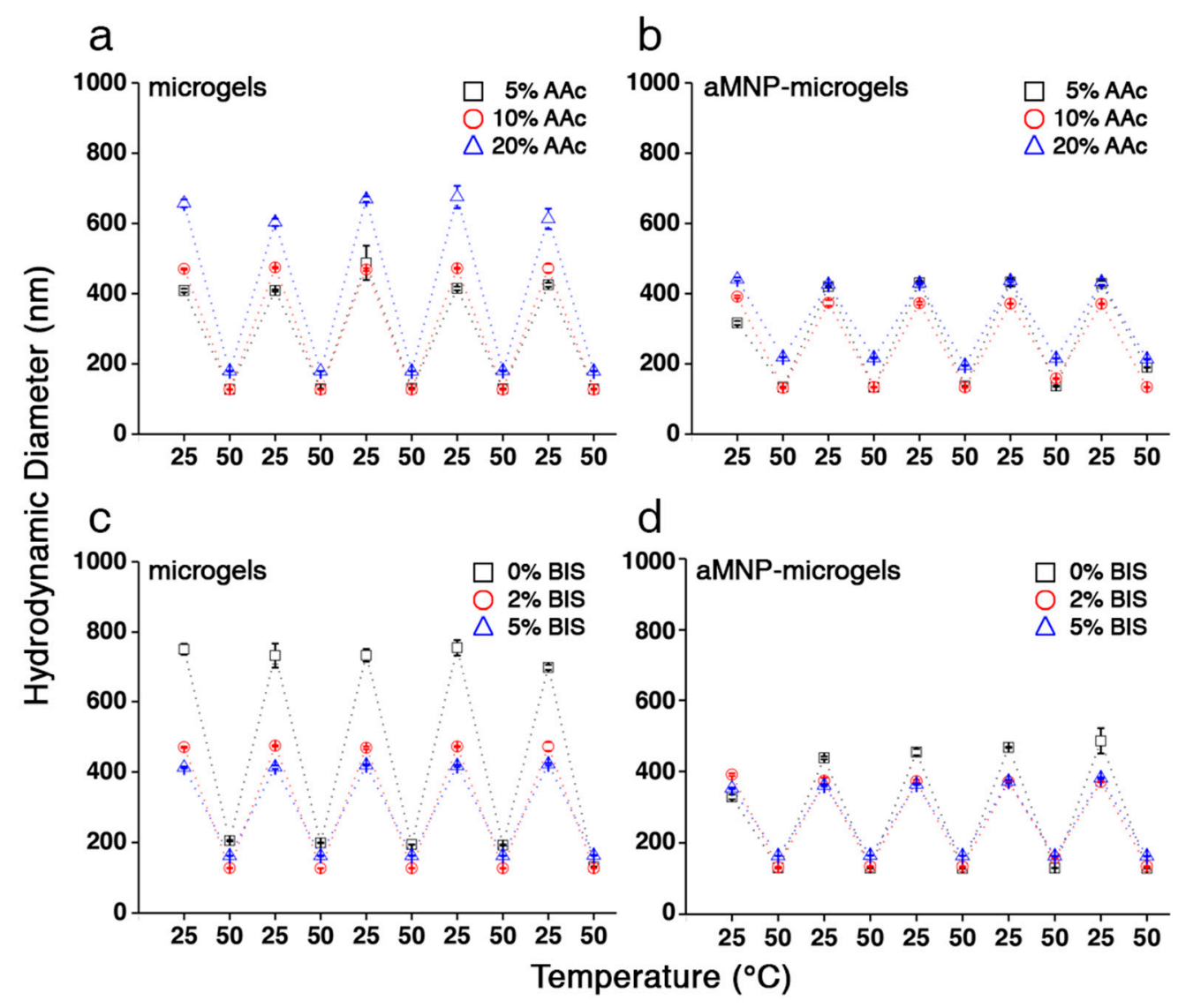

Figure 6. The hydrodynamic diameter $(\mathrm{nm})$ of $5 \%(\square), 10 \%(\bigcirc)$, and $20 \%(\triangle)$ AAc microgels without (a) and with (b) aMNP complexation and $0 \%(\square), 2 \%(\bigcirc)$, and $5 \%(\triangle)$ BIS microgels without (c) and with (d) aMNP complexation at $25^{\circ} \mathrm{C}$ and $50{ }^{\circ} \mathrm{C}$ in cooling and heating cycles, respectively. Note that each measurement was performed after $15 \mathrm{~min}$ of equilibration time at the cycles.

Similarly, thermoresponsivity of the microgels with various BIS content $(0 \%, 2 \%, 5 \%)$ is evaluated in the same temperature cycle mode, as shown in Figure 6c. The higher BIS content in microgels, the smaller the Dh at $25{ }^{\circ} \mathrm{C}$, resulting in the lesser change in the Dh at $50{ }^{\circ} \mathrm{C}$. The effects of BIS on the microgel thermoresponsivity are mimicking those of AAc, but in an inversed manner. Such trends are also measured in the case of aMNP-microgels, as shown in Figure 6d. Again, this finding suggests that the microgels with higher responsivity to nanoparticles are prepared with the lower BIS content. Thus, we confirm that the nanoparticle-microgel complexation is suitable to form advanced nanomaterials with the characteristic thermoresponsivity in a repeatable manner. 
To characterize the thermoresponsive behavior of microgels, we also measure the Dh of microgels at $1{ }^{\circ} \mathrm{C}$ intervals from 25 to $55^{\circ} \mathrm{C}$, which allows for the determination of the volume phase transition temperature (VPTT), as shown in Figure 7. Although the microgels with various AAc begin to undergo transition in their size at $\sim 31{ }^{\circ} \mathrm{C}$, the ending temperature of the phase transition differs by $10{ }^{\circ} \mathrm{C}$. As a result, the VPTTs of microgels are increased to 36,37 , and $41{ }^{\circ} \mathrm{C}$ for $5 \%, 10 \%$, and $20 \%$ of AAC, respectively, as shown in Figure 7a and Table 1. The complexation of aMNPs with microgels slightly alters their thermoresponsivity by increasing VPTT and beginning/ending transition temperature with $2 \sim 3{ }^{\circ} \mathrm{C}$, respectively, with the exception of the $5 \%$ AAc, as shown in Figure $7 \mathrm{~b}$. It is interesting to note that the initial size of aMNP-microgels is smaller than that of microgels alone but their deswelling yields similar size to the microgels. The results imply that maximum deswelling of microgels is coupled to their composition in the synthesis, while post-modification of microgels by aMNP complexation changes their properties including the Dh in swollen state, VPTT, and degree of transition.

The VPTTs of microgels are also evaluated by changing BIS content of the microgels, as shown in Figure 7c,d and Table 1. In contrast to the above experiments, the effect of BIS on the microgel's VPTT is not apparent, while the Dh of the microgels at $25^{\circ} \mathrm{C}$ is clearly different, as shown in Figure $7 \mathrm{c}$. The results suggest that AAc as a comonomer enables the modulation of both the VPTT and swollen degree of microgels, but BIS as a crosslinker favors the change of the swollen degree of microgels rather than the VPTT. However, the complexation of aMNP to microgels differs the VPTT by BIS content, as shown in Figure $7 \mathrm{~d}$. For instance, the VPTT of aMNP-microgels are increased to 38,40 , and $43^{\circ} \mathrm{C}$ for $0 \%, 2 \%$, and $5 \%$ of BIS, respectively, while the VPTT of microgels are retained at $\sim 37{ }^{\circ} \mathrm{C}$. It could be explained by considering the fact that amine groups of aMNP contribute to the charge balance of microgels. It is also worthwhile to note that the observed VPTT value $\left(38^{\circ} \mathrm{C}\right)$ for $5 \%$ BIS microgels $\left(10 \% \mathrm{AAc}\right.$, ) are comparable to previously reported values $\left(36.4^{\circ} \mathrm{C}\right)[39]$. Our results suggest that the higher the BIS content in microgels, the higher the VPTT of aMNP-microgels.

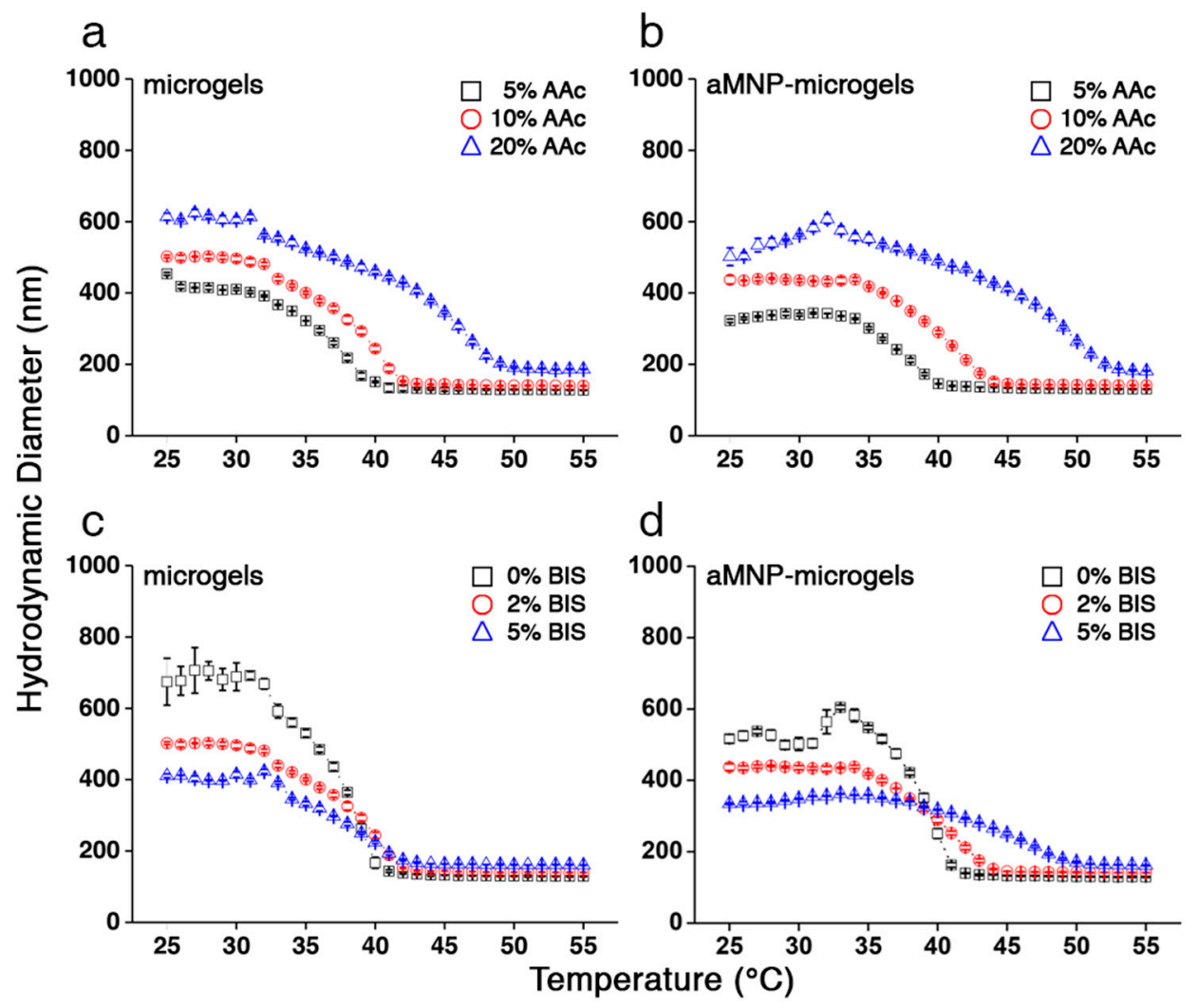

Figure 7. The hydrodynamic diameter (nm) of 5\% ( $\square), 10 \%(\bigcirc)$, and $20 \%(\triangle)$ AAc microgels without (a) and with (b) aMNP complexation and $0 \%(\square), 2 \%(\bigcirc)$, and $5 \%(\triangle)$ BIS microgels without (c) and with (d) aMNP complexation in temperature ramp from 25 to $55^{\circ} \mathrm{C}$ at one degree intervals. 
Table 1. Thermoresponsivity of microgels and aMNP-microgels via temperature ramp experiments.

\begin{tabular}{|c|c|c|c|c|c|c|c|}
\hline & & \multicolumn{3}{|c|}{ Temp $\left({ }^{\circ} \mathrm{C}\right)$} & \multicolumn{3}{|c|}{ Dh (nm) } \\
\hline & & $\begin{array}{l}\text { Transition } \\
\text { Start }\left(\mathrm{T}_{S}\right)\end{array}$ & $\begin{array}{c}\text { Transition } \\
\text { End }\left(T_{E}\right)\end{array}$ & VPTT & $\begin{array}{c}\text { Transition } \\
\text { Start }\left(\mathrm{T}_{\mathrm{s}}\right)\end{array}$ & $\begin{array}{c}\text { Transition } \\
\text { End }\left(T_{E}\right)\end{array}$ & $\mathrm{T}_{\mathrm{E}} / \mathrm{T}_{\mathrm{S}}$ \\
\hline \multirow{3}{*}{ microgels } & $5 \%$ AAc & 31 & 41 & 36 & 400 & 130 & 0.33 \\
\hline & $10 \% \mathrm{AAc}$ & 32 & 42 & 37 & 480 & 150 & 0.31 \\
\hline & $20 \%$ AAc & 32 & 50 & 41 & 560 & 190 & 0.34 \\
\hline \multirow{3}{*}{ aMNP-microgels } & $5 \%$ AAc & 34 & 41 & 38 & 330 & 140 & 0.42 \\
\hline & $10 \%$ AAc & 34 & 45 & 40 & 440 & 150 & 0.34 \\
\hline & $20 \%$ AAc & 35 & 53 & 44 & 610 & 190 & 0.31 \\
\hline \multirow{3}{*}{ microgels } & $0 \%$ BIS & 32 & 41 & 37 & 670 & 140 & 0.21 \\
\hline & $2 \%$ BIS & 32 & 42 & 37 & 480 & 150 & 0.31 \\
\hline & $5 \%$ BIS & 33 & 43 & 38 & 390 & 170 & 0.44 \\
\hline \multirow{3}{*}{ aMNP-microgels } & $0 \%$ BIS & 34 & 42 & 38 & 600 & 140 & 0.23 \\
\hline & $2 \% \mathrm{BIS}$ & 34 & 45 & 40 & 440 & 140 & 0.32 \\
\hline & $5 \%$ BIS & 35 & 51 & 43 & 360 & 170 & 0.47 \\
\hline
\end{tabular}

\section{Conclusions}

We demonstrate the thermoresponsive behavior of pNIPAm-co-AAc microgels while varying the amount of co-monomer (AAc) and crosslinker (BIS), and further complexation with aMNPs. Dynamic light scattering measurements allow for the determination of the hydrodynamic diameter of pNIPAm-co-AAc microgels that is altered not only by the cross-linking density, but also by the degree of the network charge. A method using electrostatic interaction between anionic microgels and cationic aMNP enables the formation of efficient aMNP-microgels while retaining the characteristic thermoresponsivity of the microgels. In addition, the effect of aMNP complexation on the microgels is evaluated, providing the decreased size of the microgels at $25{ }^{\circ} \mathrm{C}$ and the VPTT shifts to a higher temperature. When the aMNP-microgels are exposed to repeated cycles of heating at $50{ }^{\circ} \mathrm{C}$ (above the VPTT) and cooling at $25^{\circ} \mathrm{C}$ (below the VPTT), they display the decreased and increased hydrodynamic sizes, respectively, with no apparent hysteresis. Our results provide an example of simple and rapid modification routes that empower responsive pNIPAm-co-AAc microgels to acquire additional merits of magnetic nanoparticles for diverse applications such as biosensors, imaging agents, optical modulators, and multiresponsive smart materials.

Author Contributions: Formal Analysis/Investigation, J.K. and S.-K.L.; Writing-Original Draft Preparation, J.K. and S.-K.L.; Writing-Review \& Editing, J.K. and Y.P.; Project Administration/Conceptualization/Funding Acquisition/Supervision, J.K.

Funding: Jongseong Kim acknowledges support from National Research Foundation of Korea (NRF-2016R1D1A1B03933938) and a Korea University Grant.

Conflicts of Interest: The authors declare no conflict of interest.

\section{References}

1. Miyata, T.; Asami, N.; Uragami, T. A reversibly antigen-responsive hydrogel. Nature 1999, 399, 766-769. [CrossRef] [PubMed]

2. Kim, J.; Park, Y.; Brown, A.C.; Lyon, L.A. Direct observation of ligand-induced receptor dimerization with a bioresponsive hydrogel. RSC Adv. 2014, 4, 65173-65175. [CrossRef]

3. Kim, J.; Singh, N.; Lyon, L.A. Label-free biosensing with hydrogel microlenses. Angew. Chem. Int. Ed. Engl. 2006, 45, 1446-1449. [CrossRef] [PubMed]

4. Kim, J.; Nayak, S.; Lyon, L.A. Bioresponsive hydrogel microlenses. J. Am. Chem. Soc. 2005, 127, 9588-9592. [CrossRef] [PubMed]

5. Hong, Y.S.; Kim, J.; Sung, H.K. Characterization of a functional hydrogel layer on a silicon-based grating waveguide for a biochemical sensor. Sensors 2016, 16, 914. [CrossRef] [PubMed] 
6. Khan, F.; Tanaka, M. Designing smart biomaterials for tissue engineering. Int. J. Mol. Sci. 2018, $19,17$. [CrossRef] [PubMed]

7. Song, M.; Jang, H.; Lee, J.; Kim, J.H.; Kim, S.H.; Sun, K.; Park, Y. Regeneration of chronic myocardial infarction by injectable hydrogels containing stem cell homing factor sdf-1 and angiogenic peptide ac-sdkp. Biomaterials 2014, 35, 2436-2445. [CrossRef] [PubMed]

8. Kim, J.; Serpe, M.J.; Lyon, L.A. Hydrogel microparticles as dynamically tunable microlenses. J. Am. Chem. Soc. 2004, 126, 9512-9513. [CrossRef] [PubMed]

9. Tanaka, T.; Fillmore, D.J. Kinetics of swelling of gels. J. Chem. Phys. 1979, 70, 1214-1218. [CrossRef]

10. Dusek, K.; Patterson, K. Transition on swollen polymer networks induced by intramolecular condensation. J. Polym. Sci. Polym. Phys. Ed. 1968, 6, 1209-1216.

11. Pelton, R. Temperature-sensitive aqueous microgels. Adv. Colloid Interface Sci. 2000, 85, 1-33. [CrossRef]

12. Serpe, M.J.; Jones, C.D.; Lyon, L.A. Layer-by-layer deposition of thermoresponsive microgel thin films. Langmuir 2003, 19, 8759-8764. [CrossRef]

13. Jones, C.D.; Lyon, L.A. Synthesis and characterization of multiresponsive core-shell microgels. Macromolecules 2000, 33, 8301-8306. [CrossRef]

14. Yin, X.; Hoffman, A.S.; Stayton, P.S. Poly( $N$-isopropylacrylamide-co-propylacrylic acid) copolymers that respond sharply to temperature and ph. Biomacromolecules 2006, 7, 1381-1385. [CrossRef] [PubMed]

15. Wang, X.; Qiu, X.; Wu, C. Comparison of the coil-to-globule and the globule-to-coil transitions of a single poly( $\mathrm{N}$-isopropylacrylamide) homopolymer chain in water. Macromolecules 1998, 31, 2972-2976. [CrossRef]

16. Yoo, M.K.; Sung, Y.K.; Lee, Y.M.; Cho, C.S. Effect of polyelectrolyte on the lower critical solution temperature of poly( $N$-isopropyl acrylamide) in the poly(nipaam-co-acrylic acid) hydrogel. Polymer 2000, 41, 5713-5719. [CrossRef]

17. Gan, D.; Lyon, L.A. Tunable swelling kinetics in core-shell hydrogel nanoparticles. J. Am. Chem. Soc. 2001, 123, 7511-7517. [CrossRef] [PubMed]

18. Nayak, S.; Lyon, L.A. Soft nanotechnology with soft nanoparticles. Angew. Chem. Int. Ed. 2005, 44, 7686-7708. [CrossRef] [PubMed]

19. Kim, J.; Serpe, M.J.; Lyon, L.A. Photoswitchable microlens arrays. Angew. Chem. Int. Ed. 2005, 44, $1333-1336$. [CrossRef] [PubMed]

20. Slaughter, B.V.; Khurshid, S.S.; Fisher, O.Z.; Khademhosseini, A.; Peppas, N.A. Hydrogels in regenerative medicine. Adv. Mater. 2009, 21, 3307-3329. [CrossRef] [PubMed]

21. Boffito, M.; Sirianni, P.; Di Rienzo, A.M.; Chiono, V. Thermosensitive block copolymer hydrogels based on poly(epsilon-caprolactone) and polyethylene glycol for biomedical applications: State of the art and future perspectives. J. Biomed. Mater. Res. A 2015, 103, 1276-1290. [CrossRef] [PubMed]

22. Li, B.; Zu, S.; Zhou, J.; Jiang, Q.; Du, B.; Shan, H.; Luo, Y.; Liu, Z.; Zhu, X.; Fang, Z. Single-nanoparticle plasmonic electro-optic modulator based on $\mathrm{MoS}_{2}$ monolayers. ACS Nano 2017, 11, 9720-9727. [CrossRef] [PubMed]

23. Tseng, Y.T.; Chuang, Y.J.; Wu, Y.C.; Yang, C.S.; Wang, M.C.; Tseng, F.G. A gold-nanoparticle-enhanced immune sensor based on fiber optic interferometry. Nanotechnology 2008, 19, 345501. [CrossRef] [PubMed]

24. Shu, T.; Shen, Q.M.; Wan, Y.; Zhang, W.; Su, L.; Zhang, X.J.; Serpe, M.J. Silver nanoparticle-loaded microgel-based etalons for $\mathrm{H}_{2} \mathrm{O}_{2}$ sensing. RSC Adv. 2018, 8, 15567-15574. [CrossRef]

25. Kim, J.; Park, J.E.; Nahrendorf, M.; Kim, D.-E. Direct thrombus imaging in stroke. J. Stroke 2016, 18, $286-296$. [CrossRef] [PubMed]

26. Alkilany, A.M.; Lohse, S.E.; Murphy, C.J. The gold standard: Gold nanoparticle libraries to understand the nano-bio interface. Acc. Chem. Res. 2013, 46, 650-661. [CrossRef] [PubMed]

27. Ma, X.; Yang, Z.; Wang, Y.; Zhang, G.; Shao, Y.; Jia, H.; Cao, T.; Wang, R.; Liu, D. Remote controlling DNA hydrogel by magnetic field. ACS Appl. Mater. Interfaces 2017, 9, 1995-2000. [CrossRef] [PubMed]

28. Yata, T.; Takahashi, Y.; Tan, M.; Nakatsuji, H.; Ohtsuki, S.; Murakami, T.; Imahori, H.; Umeki, Y.; Shiomi, T.; Takakura, Y.; et al. DNA nanotechnology-based composite-type gold nanoparticle-immunostimulatory DNA hydrogel for tumor photothermal immunotherapy. Biomaterials 2017, 146, 136-145. [CrossRef] [PubMed]

29. Jiang, L.; Zhou, Q.; Mu, K.; Xie, H.; Zhu, Y.; Zhu, W.; Zhao, Y.; Xu, H.; Yang, X. Ph/temperature sensitive magnetic nanogels conjugated with cy5.5-labled lactoferrin for $\mathrm{mr}$ and fluorescence imaging of glioma in rats. Biomaterials 2013, 34, 7418-7428. [CrossRef] [PubMed] 
30. Zhao, X.-Q.; Wang, T.-X.; Liu, W.; Wang, C.-D.; Wang, D.; Shang, T.; Shen, L.-H.; Ren, L. Multifunctional au@ipn-pnipaam nanogels for cancer cell imaging and combined chemo-photothermal treatment. J. Mater. Chem. 2011, 21, 7240-7247. [CrossRef]

31. Rittikulsittichai, S.; Kolhatkar, A.G.; Sarangi, S.; Vorontsova, M.A.; Vekilov, P.G.; Brazdeikis, A.; Randall Lee, T. Multi-responsive hybrid particles: Thermo-, ph-, photo-, and magneto-responsive magnetic hydrogel cores with gold nanorod optical triggers. Nanoscale 2016, 8, 11851-11861. [CrossRef] [PubMed]

32. Laurenti, M.; Guardia, P.; Contreras-Caceres, R.; Perez-Juste, J.; Fernandez-Barbero, A.; Lopez-Cabarcos, E.; Rubio-Retama, J. Synthesis of thermosensitive microgels with a tunable magnetic core. Langmuir 2011, 27, 10484-10491. [CrossRef] [PubMed]

33. Cho, M.H.; Kim, S.; Lee, J.H.; Shin, T.H.; Yoo, D.; Cheon, J. Magnetic tandem apoptosis for overcoming multidrug-resistant cancer. Nano Lett. 2016, 16, 7455-7460. [CrossRef] [PubMed]

34. Gan, D.; Lyon, L.A. Interfacial nonradiative energy transfer in responsive core-shell hydrogel nanoparticles. J. Am. Chem. Soc. 2001, 123, 8203-8209. [CrossRef] [PubMed]

35. Jang, J.T.; Nah, H.; Lee, J.H.; Moon, S.H.; Kim, M.G.; Cheon, J. Critical enhancements of mri contrast and hyperthermic effects by dopant-controlled magnetic nanoparticles. Angew. Chem. Int. Ed. Engl. 2009, 48, 1234-1238. [CrossRef] [PubMed]

36. Kim, J.W.; Seo, D.; Lee, J.U.; Southard, K.M.; Lim, Y.; Kim, D.; Gartner, Z.J.; Jun, Y.W.; Cheon, J. Single-cell mechanogenetics using monovalent magnetoplasmonic nanoparticles. Nat. Protoc. 2017, 12, 1871-1889. [CrossRef] [PubMed]

37. Lee, S.K.; Hwang, G.; Woo, J.; Park, J.; Kim, J. Characterization of responsive hydrogel nanoparticles upon polyelectrolyte complexation. Polymers 2017, 9, 66. [CrossRef]

38. Brown, A.C.; Stabenfeldt, S.E.; Ahn, B.; Hannan, R.T.; Dhada, K.S.; Herman, E.S.; Stefanelli, V.; Guzzetta, N.; Alexeev, A.; Lam, W.A.; et al. Ultrasoft microgels displaying emergent platelet-like behaviours. Nat. Mater. 2014, 13, 1108-1114. [CrossRef] [PubMed]

39. Burmistrova, A.; Richter, M.; Eisele, M.; Uzum, C.; von Klitzing, R. The effect of co-monomer content on the swelling/shrinking and mechanical behaviour of individually adsorbed pnipam microgel particles. Polymers 2011, 3, 1575-1590. [CrossRef] 